\title{
Selenium Supplement for Obesity and Diabetes
}

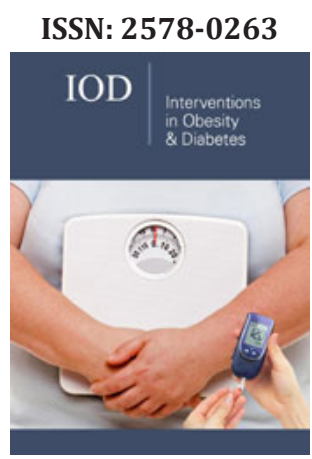

*Corresponding author: Daijie Chen, School of Pharmacy, Shanghai Jiaotong University, Shanghai, China

Submission: 此 March 06, 2020

Published: 僵 April 15, 2020

Volume 3 - Issue 5

How to cite this article: Dan Zhao, Hui Zhu, Daijie Chen. Selenium Supplement for Obesity and Diabetes. Interventions Obes Diabetes 3(5). IOD.000574. 2020. DOI: 10.31031/IOD.2020.03.000574

Copyright@Daijie Chen. This article is distributed under the terms of the Creative Commons Attribution 4.0 International License, which permits unrestricted use and redistribution provided that the original author and source are credited.

\author{
Dan Zhao, Hui Zhu, and Daijie Chen* \\ School of Pharmacy, Shanghai Jiaotong University, Shanghai, China
}

\section{Abstract}

The beneficial effects of selenium (Se) as a trace element in chronic metabolic disease had been well reported in many studies. A change of Se level in human affects body metabolism significantly. The mechanism through which the Se improves diabetes, hyperglycemia and obesity is by relieving insulin resistance, reducing appetite and upregulating genes for fatty acid breakdown. The most important effect of Se is the regulation of the immune system. The present studies reveal that Se and Se-enriched products can be considered as promising candidates for the treatment of chronic metabolic diseases especially diabetes and obesity.

Keywords: Selenium; Diabetes; Obesity; Insulin resistance; Immune regulation; Gut microbiota

Abbreviations: T2D: Type 2 diabetes; Se: Selenium; IR: Insulin Resistance; HbA1c: Glycated Hemoglobin; FBG: Fasting Blood Glucose; FBW: Fasting Body Weight; IL: Inter Leukin; TNF: Tumor Necrosis Factor; HSPs: Heat Shock Proteins; FFA: Free Fatty Acids; Se-B. longum DD98: Selenium-enriched Bifidobacterium longum DD98; AST: Alanine Amino Transferase; ALT: Aspartate Amino Transferase; CPT1: Carnitine Palmitoyl Transferase-1; PPAR $\alpha$ : Peroxisome Proliferator-Activated Receptor Alpha; FAS: Fatty Acid Synthase; LPL: Lipo Protein Lipas; PPAR $\gamma$ : Peroxisome Proliferator Activated Receptor Gamma; NAFLD: Non-Alcoholic Fatty Liver Disease

\section{Introduction}

Obesity is one of the most common metabolic disorder diseases, which is caused by multiple factors including eating habits, environment, and genetics. It is stored in the form of fat when body's energy intake exceeds consumption [1]. It is characterized by excessive accumulation and storage of fat in the body, which can accelerate the development of many chronic diseases and is a risk factor that causes diabetes. Diabetes is one of the global health problems, and type 2 diabetes (T2D), a chronic metabolic disease caused by the decrease of insulin receptor sensitivity and the increase of fasting blood glucose, accounts for about $90 \%$ of diabetes [2]. Selenium (Se) is an essential trace element in human diet. Previous studies had reported the effect of Se as a dietary supplement in yogurt [3]. Se exerts its bioactivities mainly through selenoproteins and the functions of selenoproteins strictly depend on the presence of selenocysteine, which is the $21^{\text {st }}$ "naturally occurring" amino acid in the genetic code and is largely responsible for the health-promoting effects of Se. There are 25 selenoproteins in human body, and the physiological functions of some selenoproteins have been wellcharacterized [4]. Se plays an important role in human immune function, which is closely related to the development of obesity and T2D. Refer to the average dietary micronutrient requirements of Chinese residents, the proportions of men and women with insufficient selenium intake was $49.6 \%$ and $65.4 \%$, respectively. Long-term Se deficiency significantly suppresses the immune response, reduces disease resistance, and increases the risk of chronic metabolic disease such as diabetes and obesity [5]. Additionally, selenoproteins are central for antioxidant system regulation in human body [6], and protects the human liver and other organs from oxidative stress injury caused by obesity and diabetes. Furthermore, Se also improves the chronic metabolic diseases via influencing the composition of gut microbiota [7]. Supplementation of Se is a good therapeutic strategy to regulate the immune system, relieve the oxidative stress and maintain the intestinal homeostasis, thereby improving the physiological functions of the host. This review mainly discusses about the beneficial effects of Se in chronic metabolic disease, especially in T2D and obesity.

\section{Discussion}

Se has been proved to have multiple biological functions in chronic metabolic disease, such as antioxidation, antitumor, improving the balance of lipid metabolism, and regulating the immune system $[8,9]$. In T2D, Se can directly reduce the symptoms of diabetes, especially the 
fasting blood glucose (FBG) levels, through reversing the changes of some basic biochemical indicators, such as glycated hemoglobin (HbA1c) and leptin [10,11]. Additionally, Se may relieve the impaired glucose and insulin tolerance, and thereby improving the insulin sensitivity and lowering the blood glucose levels radically [12]. Leptin is a protein hormone secreted by adipose tissue, which can reduce food intake, increase energy release, inhibit the synthesis of fat cells, and ultimately reduce weight [13]. On the other hand, Se reduces fasting boy weight (FBW) in patients with obesity mainly by regulating not only the serum leptin but also the leptin in adipose tissue [14]. Se (especially selenoprotein) exhibits notable anti-inflammatory capacity in the host, which is closely related to the immune function [15]. Se can regulate the levels of some pro-inflammatory factors, especially the IL-1, IL- 6 and TNF- $\alpha$, through the NF- $\kappa B$ pathway, which is an important pathway for the regulation of inflammatory factors [16]. Some findings had presented that the high levels of pro-inflammatory factors induced the pancreatic injuries, which subsequently caused insufficient insulin secretion or decreased insulin sensitivity, eventually forming insulin resistance (IR) and triggering diabetes $[17,18]$. Moreover, the direct inhibition effect of inflammatory factors for insulin signaling pathway had been reported [19]. Therefore, Se exerts beneficial effects on T2D via reducing inflammation, alleviating pancreas damage, improving IR, and relieving the diabetes effectively and fundamentally. Furthermore, Se can improve human immunity by relieving lymphocytes injury caused by obesity. Lymphocytes are important components of the immune response, while obesity induces inflammation, decreases immune function and eventually causes serious injury in the lymphocytes. However, Se can suppress obese damage to lymphocytes by reducing the extensive high levels of heat shock proteins (HSPs) [20]. In addition, previous studies had shown that Se could reduce oxidative stress and inflammatory response, remedying the organism in serious state of stress $[21,22]$. A recent paper showed that the Se and its therapeutic effectiveness on chronic metabolic disease may be linked to activation of Sirt 1 , which may increase acetylation of $\mathrm{p} 53$, activate protein NF- $\kappa B$ and is critical for pro-inflammatory response [23]. In summary, Se significantly improves chronic metabolic diseases such as obesity or diabetes by suppressing inflammation and improving immunity in humans.

The change of free fatty acids (FFA) in serum is an important indicator for lipid metabolism disorders. The pro-obesity effect of high levels of FFA in serum is well known. Also, high concentration of FFA in serum may reduce peripheral tissue sensitivity to insulin and decrease glucose uptake, triggering IR [24-26]. The secretion of inflammation factors in obesity can inhibit the synthesis of triglycerides, promote lipolysis and increase the level of FFA in serum, which in turn causes IR and triggers diabetes eventually $[27,28]$. Above discussion has stated the anti-inflammation effect of Se in chronic metabolic diseases. Therefore, Se can inhibit lipolysis and the FFA levels indirectly through suppressing the secretion of pro-inflammatory factors, which would improve IR and relieve diabetes. Also, Se can decrease the level of FFA in serum with the same mechanism, which directly improves the symptoms of obesity. In addition, previous study has indicated that FFA levels are related to the increase of oxidative stress [29]. Oxidative stress induces continuous oxidative damages in animal cells, which increases the risk of hepatic steatosis, vascular and heart disease by disrupting the antioxidant balance in the human body [30]. Se can decrease the oxidative stress by regulating the levels of alanine aminotransferase (AST) and aspartate aminotransferase (ALT) to protect against the liver damage induced by excess oxidative stress accumulation happened in diabetes and obesity [31]. According to report, Se significantly increased the plasma antioxidant capacity by $42 \%$ in obese mice [32]. Notably, in that study, Se has a huge effect in the production of glutathione peroxidase, and a little effect on the level of superoxide dismutase. Moreover, T2D and obesity are main clinical manifestations of metabolic syndrome, while non-alcoholic fatty liver disease (NAFLD) is hepatic manifestation of metabolic syndrome. Nowadays, given the prevalence of metabolic syndrome, nonalcoholic fatty liver disease has been the most common cause of liver disease in society. A study in obese rats again confirmed that $\mathrm{Se}$, whose antioxidant properties has been proven in the above discussion, may reduce and prevent damaging effects of fatty liver in rats by decreasing the excessive serum levels of total cholesterol, triglyceride, AST and ALT [33]. In summary, Se can suppress the accumulation of oxidative stress, thereby avoiding damages to organs in chronic metabolic diseases, which is critical for the prevention and therapy of predicated global NAFLD epidemic. High fat diet significantly decreases the expression of genes involved in fatty acid oxidation and increases the expression of genes involved in the regulation of adipogenesis and lipogenesis. And obesity is mainly due to the increase of adipose tissue lipogenic gene expression and the decrease of lipolytic gene expression [34]. A study in obese mice treated with Se showed that Se prevented dietinduced obesity, possibly via the mechanism of regulating lipolytic gene expression and lipogenic gene expression. Se can up-regulate mRNA expression of carnitine palmitoyltransferase-I (CPT1) and peroxisome proliferator-activated receptor alpha (PPAR $\alpha$ ), and down-regulated mRNA expression of fatty acid synthase (FAS), lipoprotein lipase (LPL), and peroxisome proliferator-activated receptor gamma (PPAR $\gamma$ ) involved in lipid metabolism [32]. Generally speaking, diabetes is also accompanied by serum lipid disorders, which increases the risk of cardiovascular complications. Another in-vivo experiment indicated that Se supplementation can obviously reverse the changes of total cholesterol, triglyceride, lowdensity lipoprotein, total protein and high-density lipoprotein levels in serum [35]. Therefore, Se may reduce the risk of cardiovascular complications by balancing lipid metabolism in diabetes. In addition, recent reports presented that such beneficial effect of Se is related to activation of Sirt 1, which can inhibit PPAR $\gamma$-mediated fat differentiation, promote fat metabolism, and lower blood lipids [23].

The gut microbiota is a complex ecosystem, which plays a vital role in regulating human physiological and metabolic functions. At present, the mechanism of intestinal flora to regulate diabetes and obesity mainly includes the following aspects. First of all, the type and proportion of intestinal flora can directly affect obesity or T2D [36]. Secondly, gut flora can relieve obesity by regulating host's immune system [37]. Thirdly, Short-chain fatty acids secreted by 
intestinal microorganisms can improve metabolism and epithelial barrier integrity in many ways [36]. There are also other regulatory mechanisms of the intestinal flora, such as intestinal florahypothalamus-mechanism of obesity regulation and so on. Previous reports demonstrated that the composition of the gut microbiota in mice influenecd the host Se levels, and that the Se levels also could alter the composition of the gut microbiota [38]. The diversity of the intestinal bacteria increased in animals fed with higher levels of Se. Se affects both the composition of the intestinal microbiota and the colonization of the gastrointestinal tract, which, in turn, influence the Se state and selenoproteome expression of the host [7]. Hence, Se and selenoproteins are closely related to gastrointestinal health and protective effects of Se in chronic metabolic disease could be mediated via a Se-gut microbiota pathway. Furthermore, studies have confirmed the synergistic effect of Se and probiotics in chronic metabolic diseases [32]. Our laboratory also developed a Se-enriched probiotic, Se-enriched Bifidobacterium longum DD98 (Se- Bifidobacterium longum DD98). Based on our results (data unpublished), Se- Bifidobacterium longum DD98 showed significant beneficial effects on obesity and T2D, and exhibited greater anti-diabetic and anti-obese effects compared with single Se or probiotics treatment. This superior function of Se-enriched probiotics could be attributed to the combination effects of Se and probiotics.

\section{Conclusion}

Many studies in animals and humans proved the important roles of Se in relieving chronic metabolic disease, such as T2D and obesity. The beneficial effects of Se are primarily mediated by suppressing inflammatory response, improving immune system, alleviating oxidative stress, and maintaining the intestinal hemostasis in the host. Se usually works as a dietary supplement or in combination with other substances, and Se-enriched probiotics could be considered as a promising candidate for the treatment of T2D and obesity. However, the application of Se in chronic metabolic diseases still requires more human trails to further confirm its therapeutic efficacy and toxicity.

\section{References}

1. Hulston CJ,Churnside AA, Venables MC (2015) Probiotic supplementation prevents high-fat, overfeeding-induced insulin resistance in human subjects. British Journal of Nutrition 113(4): 596-602.

2. Charu A, Kashish B, Mukta P, Kanika S, Varsha C, et al. (2019) Association of coagulation profile with microvascular complications and glycemic control in type 2 diabetes mellitus-A study at a tertiary care center in Delhi. Hematology Transfusion \& Cell Therapy 41(1): 31-36.

3. Kubachka KM, Hanley T, Mantha M, Wilson RA, Caruso J, et al. (2016) Evaluation of selenium in dietary supplements using elemental speciation. Food Chemistry 218: 313-320.

4. Fairweather Tait SJ, Bao Y, Broadley MR, Collings R, Ford D, et al. (2011) Selenium in human health and disease. Antioxidants \& Redox Signaling 14(7): 1337-1383.

5. Liu T, Yang T, Pan T, Liu C, Li S (2017) Effect of low-selenium/high-fat diet on pig peripheral blood lymphocytes: Perspectives from selenoproteins, heat shock proteins, and cytokines. Biological Trace Element Research 183(1):102-113.
6. Behne D, Alber D, Kyriakopoulos A (2010) Long-term selenium supplementation of humans: Selenium status and relationships between selenium concentrations in skeletal muscle and indicator materials. Journal of Trace Elements in Medicine \& Biology 24(2): 99-105.

7. Kasaikina MV, Kravtsova MA, Lee BC, Seravalli J, Peterson DA, et al. (2011) Dietary selenium affects host selenoproteome expression by influencing the gut microbiota. FASEB J 25(7): 2492-2499.

8. Qian Shen, Bowen Zhang, Rihua Xu, Wang Yang, Xuelong Ding, et al. (2010) Antioxidant activity in vitro of the selenium-contained protein from the Se-enriched Bifidobacterium animalis 01. Anaerobe 16(4): 380386.

9. Jacobs ET, Lance P, Mandarino LJ, Ellis NA, Chow HS, et al. (2019) Selenium supplementation and insulin resistance in a randomized, clinical trial. BMJ Open Diabetes Research \& Care.

10. Rayman MP, Winther KH, Pastor Barriuso R, Cold F, Thvilum M, et al. (2018) Effect of long-term selenium supplementation on mortality: results from a multiple-dose, randomised controlled trial. Circulation 135(1).

11. Tanko Y, Jimoh A, Ahmed A, Adam A, Ayo JO (2017) Effects of selenium yeast on blood glucose and antioxidant biomarkers in cholesterol fed diet induced type 2 diabetes mellitus in wistar rats. Nigerian Journal of Physiological Sciences 31(2): 147-152.

12. Liu F, Celi P, Cottrell JJ, Chauhan SS, Leury BJ, et al. (2018) Effects of a short-term supranutritional selenium supplementation on redox balance, physiology and insulin-related metabolism in heat-stressed pigs. Journal of Animal Physiology \& Animal Nutrition 102(1): 276-285.

13. Stefano JT, Torres MM, Pereira IVA, Jimenez D, Oliveira CP, et al. (2015) P0922: The combination of probiotics and prebiotics supplementation improves lipid metabolism, NAFLD and obesity in ob/ob mice. Journal of Hepatology 62(2): S691.

14. Gong T, Torres DJ, Berry MJ, Pitts MW (2018) Hypothalamic redox balance and leptin signaling - Emerging role of selenoproteins. Free Radical Biology \& Medicine 127:172-181.

15. Yao H, Zhao W, Zhao X, Fan R, Khoso PA, et al. (2014) Selenium deficiency mainly influences the gene expressions of antioxidative selenoproteins in chicken muscles. Biological Trace Element Research 161(3): 318-327.

16. Li D, Fu Y, Zhang W, Su G, Liu B, et al. (2013) Salidroside attenuates inflammatory responses by suppressing nuclear factor- $\mathrm{\kappa} B$ and mitogen activated protein kinases activation in lipopolysaccharide-induced mastitis in mice. Inflammation Research 62(1): 9-15.

17. Eguchi K, Manabe I (2013) Macrophages and islet inflammation in type 2 diabetes. Diabetes, Obesity and Metabolism 15(3): 152-158.

18. Cani PD, Everard A (2014) Akkermansia muciniphila: A novel target controlling obesity, type 2 diabetes and inflammation. Medecine Sciences 30(2): 125-127.

19. Jorgensen SB, O Neill HM, Sylow L, Honeyman J, Steinberg GR, et al. (2012) Deletion of skeletal muscle SOCS3 prevents insulin resistance in obesity. Diabetes 62(1): 56-64.

20. Dong Yu, Ziwei Zhang, Haidong Yao, Shu Li, Shiwen Xu (2015) The role of selenoprotein $\mathrm{W}$ in inflammatory injury in chicken immune tissues and cultured splenic lymphocyte. BioMetals 28(1): 75-87.

21. Reddi AS, Bollineni JS (2001) Selenium-deficient diet induces renal oxidative stress and injury via TGF-1 in normal and diabetic rats. Kidney International 59(4): 1342-1353.

22. Yang T, Zhao Z, Liu T, Zhang Z, Wang P, et al. (2017) Oxidative stress induced by Se-deficient high-energy diet implicates neutrophil dysfunction via Nrf2 pathway suppression in swine. Oncotarget 8(8): 13428-13439.

23. Cosín Tomàs $M$, Senserrich J, Arumí Planas $M$, Alquézar C, Pallàs $M$, et al. (2019) Role of resveratrol and selenium on oxidative stress 
and expression of antioxidant and anti-aging genes in immortalized lymphocytes from alzheimer's disease patients. Nutrients 11(8): E1764.

24. Gonzalez Franquesa A, Patti ME (2017) Insulin resistance and mitochondrial dysfunction. Adv Exp Med Biol 982: 465-520.

25. Karpe F, Dickmann JR, Frayn KN (2011) Fatty acids, obesity, and insulin resistance: Time for a reevaluation. Diabetes 60(10): 2441-2449.

26. Wang YY, Zhang J, Chuan Cui LU, Meng JL (2017) Clinical application of detection of superoxide dismutase and free fatty acids in patients with insulin resistance. Labeled Immunoassays \& Clinical Medicine.

27. Han J, Meng Q, Shen L, Wu G (2018) Interleukin-6 induces fat loss in cancer cachexia by promoting white adipose tissue lipolysis and browning. Lipids in Health \& Disease 17(1): 14.

28. Nguyen KD, Qiu Y, Cui X, Goh YPS, Mwangi J, et al. (2011) Alternatively activated macrophages produce catecholamines to sustain adaptive thermogenesis. Nature 480(7375): 104-108.

29. Zhang X, Yanfeng Wu, Wang Y, Wang X, Piao C, et al. (2017) The protective effects of probiotic-fermented soymilk on high-fat dietinduced hyperlipidemia and liver injury. Journal of Functional Foods 30: 220-227.

30. Masaru Kunitomo (2007) Oxidative stress and atherosclerosis. Yakugaku Zasshi Journal of the Pharmaceutical Society of Japan 127(12): 19972014.

31. Xia SF, Xie ZX, Qiao Y, Li LR, Cheng XR, et al. (2014) Salvianolic acid B counteracts cognitive decline triggered by oxidative stress in mice fed with high-fat diets. Journal of Functional Foods 11: 278-292.
32. Nido SA, Shituleni SA, Mengistu BM, Liu Y, Khan AZ, et al. (2016) Effects of selenium-enriched probiotics on lipid metabolism, antioxidative status, histopathological lesions, and related gene expression in mice fed a high-fat diet. Biological Trace Element Research 171(2): 399-409.

33. Hossein KJ, Faezeh S, Mohsen M (2017) Examining the effect of selenium in improving nonalcoholic fatty liver disease in rats. Journal of Liver 6: 2.

34. Haibo Zhang, Xiangfei Zhang, ZhiSheng Wang, Xianwen Dong, Cui Tan, et al. (2015) Effects of dietary energy level on lipid metabolism-related gene expression in subcutaneous adipose tissue of Yellow breed $\times$ Simmental cattle. Animal Science Journal 86(4): 392-400.

35. Clark LC, Combs GF, Turnbull BW, Slate EH, Chalker DK, et al. (1996) Effects of selenium supplementation for cancer prevention in patients with carcinoma of the skin. Jama 276(24): 1957-1963.

36. Xiangfei Li, Xu Q Tian Jiang, Shuguang Fang, Guang Wang, et al. (2016) A comparative study of the antidiabetic effects exerted by live and dead multi-strain probiotics in the type 2 diabetes model of mice. Food \& Function 7(12): 4851-4860.

37. Yuancheng Si, WeiNa Miao, JiaYue He, Xiaofang Wang, Yi Li Wang, et al. (2016) Intricate interactions of obesity, intestinal flora and toll-like receptors. World Chinese Journal of Digestology 24(15): 2361.

38. Hrdina J, Banning A, Kipp A, Loh G, Blaut M, et al. (2009) The gastrointestinal microbiota affects the selenium status and selenoprotein expression in mice. J Nutr Biochem 20(8): 638-648. 